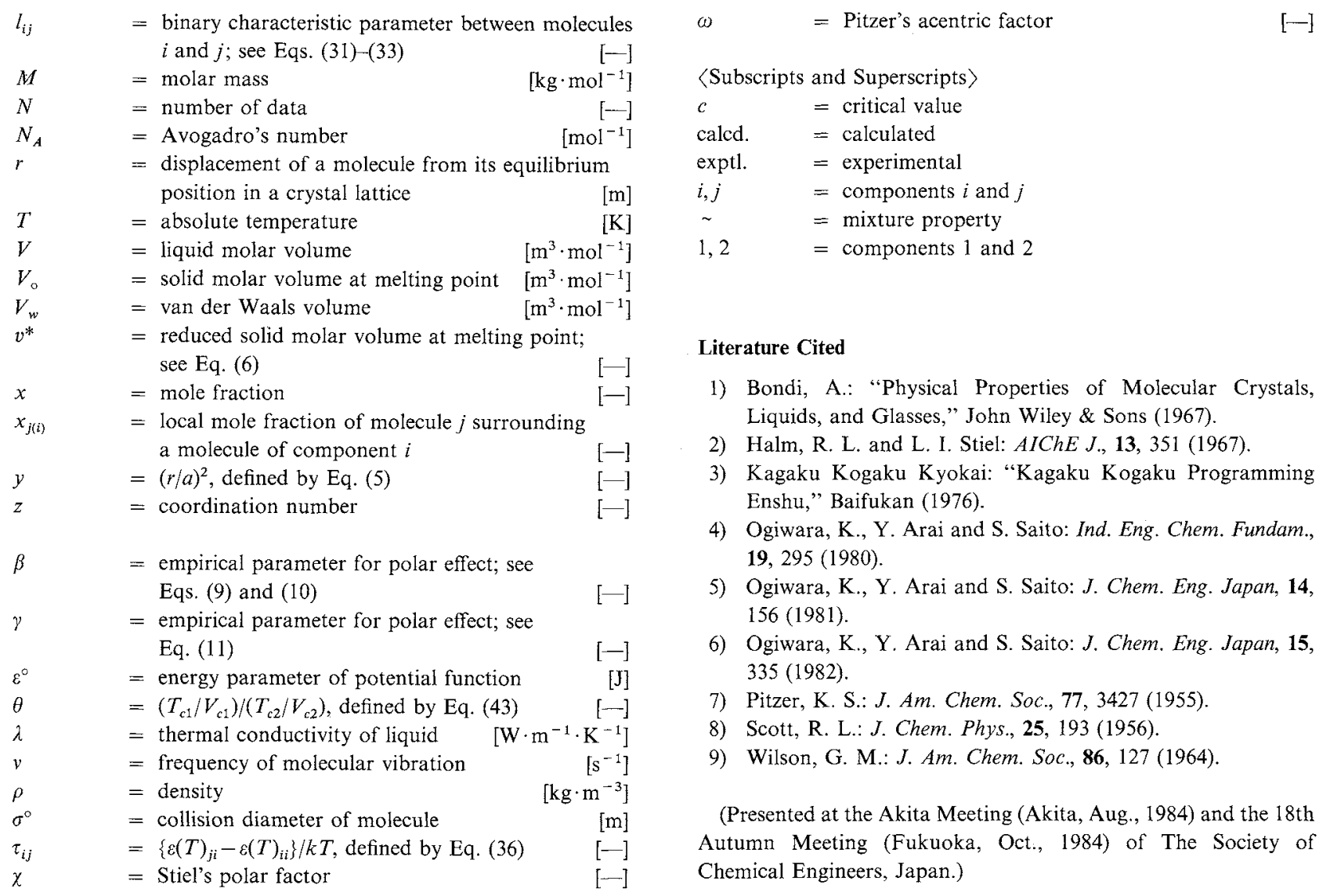

\title{
HIGH-PRESSURE VAPOR-LIQUID EQUILIBRIUM CALCULATIONS BY USE OF THE CORRESPONDING-STATES PRINCIPLE
}

FUAN-NAN TSAI AND JANN-HWA SHYU

Department of Chemical Engineering, National Cheng Kung University, Tainan, Taiwan 700

Key Words: Vapor-Liquid Equilibrium, Hydrocarbon, Corresponding-States Principle

Prediction of vapor-liquid equilibria of asymmetric mixtures by use of the corresponding-states principle has recently been developed by a number of investigators. ${ }^{1,3,6)} \mathrm{J} \mathrm{ffe}^{3)}$ and Plöcker et al. ${ }^{6)}$ have used the Lee-Kesler correlations ${ }^{4)}$ with the pseudocritical method. Arai et al. ${ }^{11}$ presented mixing rules combining functionally different equations of state on the basis of the three-parameter corresponding-states principle.

Received September 27,1984. Correspondence concerning this article should be addressed to F.-N. Tsai.
In this work we use a one-fluid theory with new mixing rules on the basis of the corresponding-states principle to calculate the vapor-liquid equilibria for asymmetric mixtures of hydrocarbons. Data used in evaluating this calculation are taken from the literature.

\section{Corresponding-States Principle with Mixing Rules}

Following Van Ness, ${ }^{10}$ ) the fugacity coefficient of component $i$ at constant $T$ and $P$ is related to the mixture fugacity coefficient by 


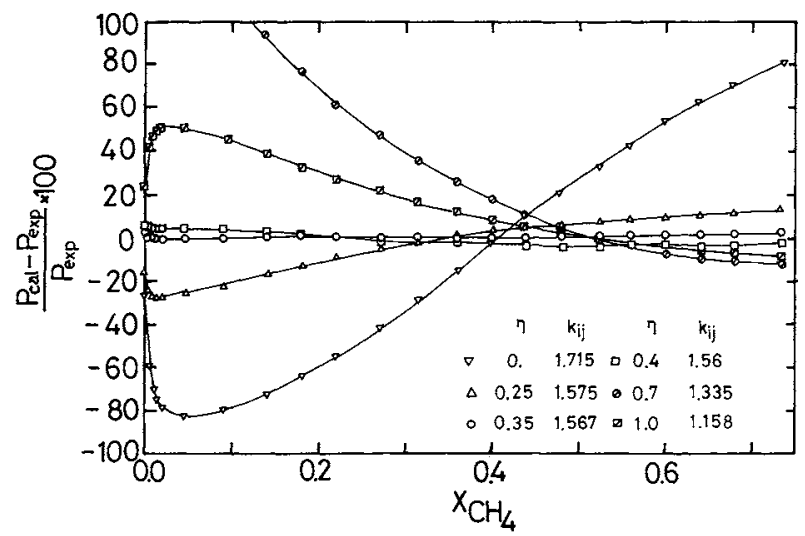

Fig. 1. Deviations between calculated and experimental bubble pressures for the binary system methane $n$-decane at $444.26 \mathrm{~K}^{7)}$ for different values of $\eta$.

$$
\begin{aligned}
\ln \phi_{i}=\ln \phi_{M} & -\frac{1}{R T} \frac{\Delta h_{M}}{T_{C M}} \sum_{j \neq i} z_{j}\left(\frac{d T_{C_{M}}}{d z_{j}}\right)_{z_{k}} \\
& +\frac{Z_{M}-1}{P_{C M}} \sum_{j \neq i} z_{j}\left(\frac{d P_{C M}}{d z_{j}}\right)_{z_{k}} \\
& -\left(\frac{\partial \ln \phi_{M}}{\partial \omega_{M}}\right)_{T_{r}, P_{r}} \sum_{j \neq i} z_{j}\left(\frac{d \omega_{M}}{d z_{j}}\right)_{z_{k}} \quad(k \neq i, j)
\end{aligned}
$$

where $\ln \phi_{M},\left(\Delta h / R T_{C}\right)_{M}$ and $Z_{M}$ are obtained from the Teja correlation ${ }^{9}$ :

$$
g_{M}\left[T_{r}, P_{r}, \omega_{M}\right]=\sum_{i}^{n} z_{i} g^{(i)}\left[T_{r}, P_{r}, \omega_{i}\right]
$$

To calculate $\ln \phi_{M},\left(\Delta h / R T_{C}\right)_{M}$ and $Z_{M}$ we use a one-fluid theory. The pseudocritical method, similar to that of Plöcker et al., ${ }^{6)}$ is used here, and a set of mixing rules for the averaging procedure to calculating the pseudocritical constants of a mixture is proposed as follows:

$$
\begin{aligned}
& T_{C M} v_{C M}^{\eta}=\sum_{i} \sum_{j} z_{i} z_{j} T_{c i j} v_{c i j}^{\eta} \\
& v_{C M}=\sum_{i} \sum_{j} z_{i} z_{j} v_{c i j} \\
& T_{c i j} v_{c i j}^{\eta}=k_{i j}\left[\left(T_{c i} T_{c j}\right)\left(v_{c i} v_{c j}\right)^{\eta}\right]^{1 / 2} \\
& v_{c i j}=\frac{1}{8}\left(v_{c i}^{1 / 3}+v_{c j}^{1 / 3}\right)^{3} \\
& P_{C M}=\left(0.2905-0.085 \omega_{M}\right) R T_{C M} / v_{C M} \\
& \omega_{M}=\sum z_{i} \omega_{i}
\end{aligned}
$$

\section{Calculation Procedure}

The calculation procedure for vapor-liquid equilibria is similar to that of Plöcker et $a l^{6)}$ and Arai et al. ${ }^{1)}$

\section{Results and Discussion}

The mixing rules proposed in this work are similar
Table 1. Binary parameter $k_{i j}$ for binary vapor-liquid equilibria

\begin{tabular}{lcccc}
\hline \multicolumn{1}{c}{ System } & $\begin{array}{c}\text { Temperature } \\
\text { range } \\
{[\mathrm{K}]}\end{array}$ & $\begin{array}{c}\text { Max. } \\
\text { pressure } \\
\text { [atm] }\end{array}$ & $\begin{array}{c}\text { Set of } \\
\text { eqs. }\end{array}$ & $k_{i j}$ \\
\hline Methane-ethane & $130-200$ & 51 & a-b & 1.049 \\
Methane-propane & $130-214$ & 64 & a-b & 1.113 \\
Methane- $n$-butane & $166-283$ & 113 & a-b & 1.185 \\
Methane-isobutane & $311-378$ & 109 & a-b & 1.160 \\
Methane- $n$-pentane & $311-444$ & 167 & a-b & 1.267 \\
Methane- $n$-hexane & $323-423$ & 160 & a-b & 1.315 \\
Methane- $n$-octane & $298-423$ & 70 & a-b & 1.448 \\
Methane- $n$-decane & $378-511$ & 336 & a-b & 1.567 \\
Methane- $n$-hexadecane & $462-623$ & 249 & a-b & 1.870 \\
Methane-toluene & $222-255$ & 136 & a-b & $1: 335$ \\
Methane-diphenylmethane & $462-623$ & 250 & a-b & 1.652 \\
Ethane-propane & $200-255$ & 15 & b-b & 1.020 \\
Ethane- $n$-pentane & $278-411$ & 67 & b-b & 1.072 \\
Ethane- $n$-octane & $313-373$ & 52 & b-b & 1.170 \\
Propane- $n$-butane & $363-393$ & 40 & b-b & 1.002 \\
Propane- $n$-pentane & $344-444$ & 44 & b-b & 1.020 \\
Propane- $n$-hexane & $363-453$ & 48 & b-b & 1.040 \\
Propane- $n$-heptane & $363-473$ & 48 & b-b & 1.070 \\
Propane- $n$-decane & $278-511$ & 70 & b-b & 1.153 \\
$n$-Butane- $n$-decane & $311-511$ & 49 & b-b & 1.090 \\
Ethylene-propylene & $263-293$ & 52 & a-b & 1.015 \\
Ethylene-1-butene & $273-293$ & 54 & a-b & 1.037 \\
\hline & & & & \\
\hline
\end{tabular}

* a: Bender's equation of state. ${ }^{2)}$ b: Generalized Benedict-Webb-Rubin equation of state. ${ }^{5}$

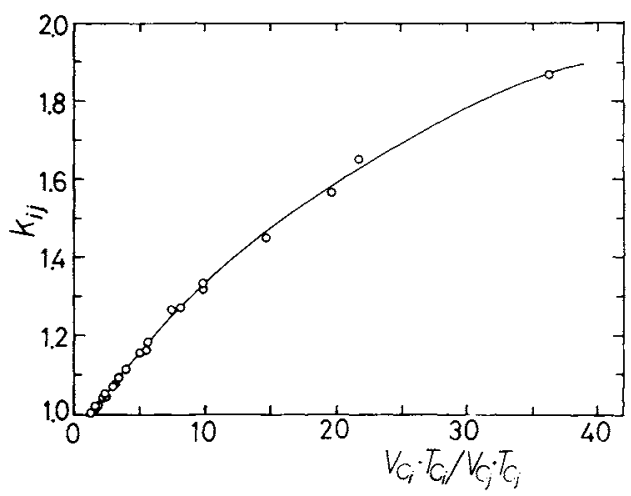

Fig. 2. Correlation of binary parameters $k_{i j}$ for hydrocarbon-hydrocarbon systems.

to those presented by a number of authors. ${ }^{1,3,4,6)}$ From the computed results it can be found that the exponent $\eta$ appearing in Eqs. (3) and (5) is of no importance for slightly asymmetric mixtures, that is, the calculated deviations of bubble pressure and vapor composition change slightly as the values of $\eta$ change from zero to unity. However, for strongly asymmetric mixtures the exponent $\eta$ becomes significant, as shown in Fig. 1. We have determined $\eta$ empirically by a systematic study of numerous asymmetric mixtures of hydrocarbons. The calculated results show that the best value of $\eta$ changes somewhat from one system to another, and good agreement with experiment is obtained by setting $\eta=0.35$ for all 
Table 2. Comparison of experimental and calculated vapor-liquid equilibria using different methods

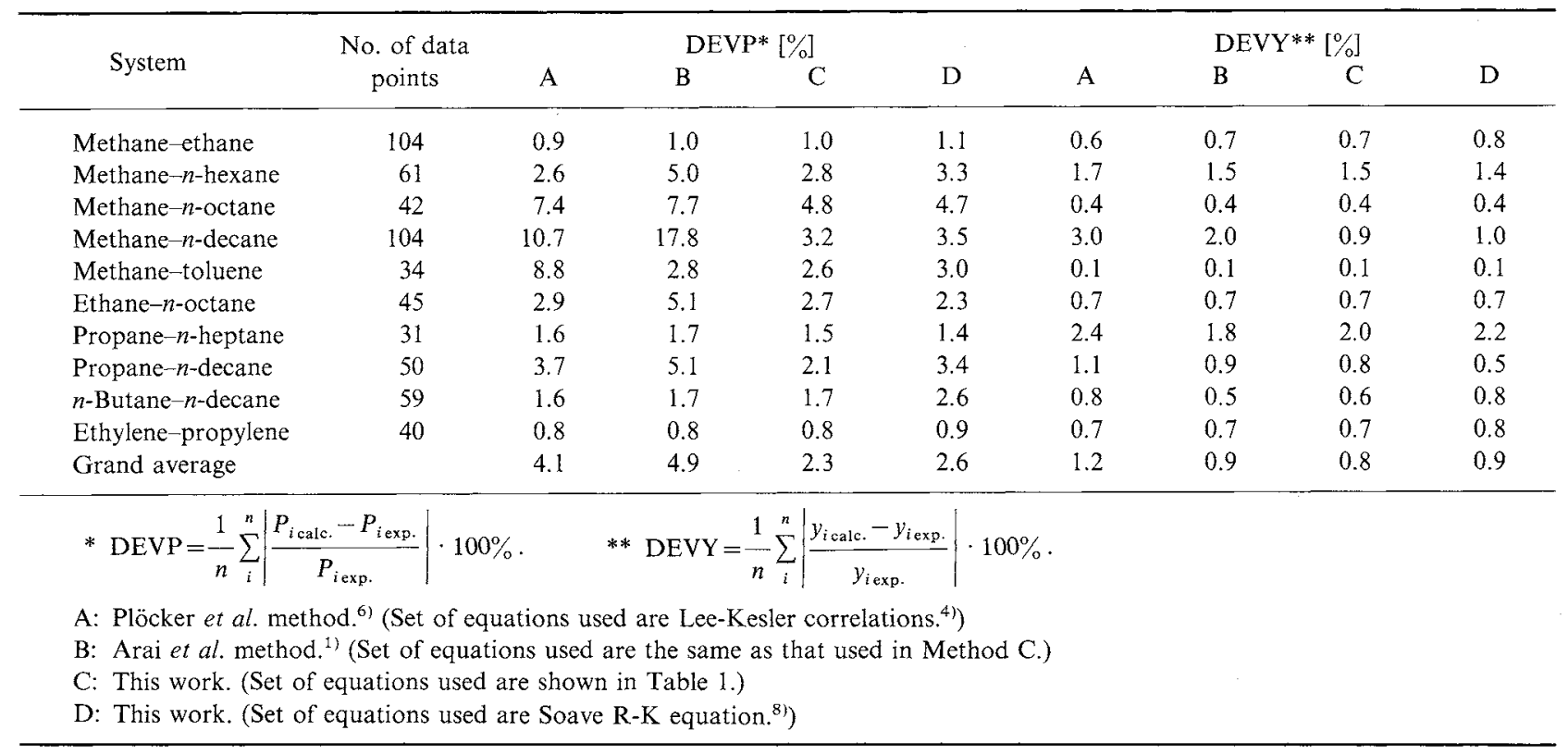

binary systems studied in this work.

Vapor-liquid equilibrium calculations are performed for 22 binary hydrocarbon-hydrocarbon systems. The obtained best values of $k_{i j}$ are listed in Table 1. Figure 2 shows $k_{i j}$ as a function of the dimensionless group $\left(T_{c i} v_{c i}\right) /\left(T_{c j} v_{c j}\right)$ and the correlation can be approximately expressed by

$k_{i, j}=0.945989+0.042214\left(\frac{T_{c i} v_{c i}}{T_{c j} v_{c j}}\right)-0.000467\left(\frac{T_{c i} v_{c i}}{T_{c j} v_{c j}}\right)^{2}$

Equation (9) can be used to estimate $k_{i j}$ value of other hydrocarbon-hydrocarbon systems where insufficient experimental information is at hand.

Table 2 compares the deviations in calculated and experimental bubble pressure and vapor composition between those obtained by Plöcker et al ${ }^{6)}$ and Arai et $a l .{ }^{1)}$ and those obtained from the methods proposed here by using the BWR type equation and Soave-RK equation. It can be seen that the calculated results do not differ appreciably for symmetric and slightly asymmetric systems. However, for strongly asymmetric systems, both methods proposed here are superior.

Vapor-liquid equilibria for the ternary system can be calculated by using binary parameters listed in Table 1. Figure 3 compares calculated $K$ values of the strongly asymmetric system methane-propane- $n$ decane with experimental data. The calculated $K$ values of methane and propane agree very well with experimental data, and the calculated $K$ values of $n$ decane also agree well with experimental data except for the high-pressure region.

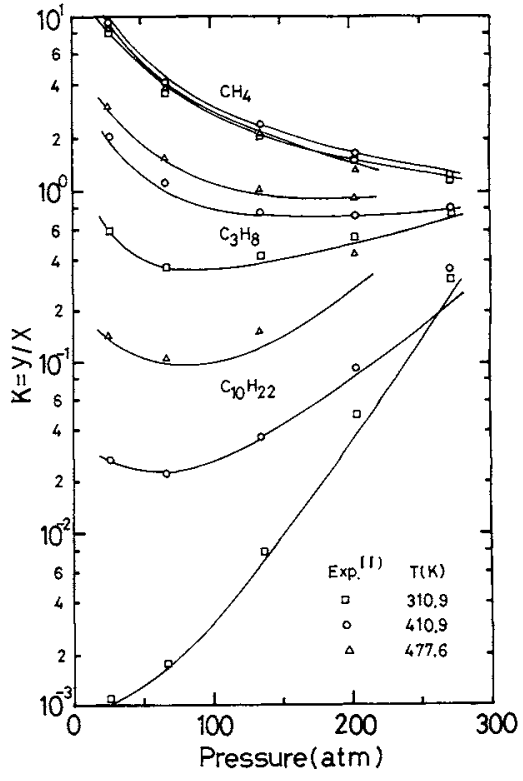

Fig. 3. Calculated $K$ values for the ternary system methane-propane $n$-decane; comparison with experimental data of Wiese et al. ${ }^{11)}$

Nomenclature
$\left.\begin{array}{llr}g & =\text { thermodynamic property } \\ \Delta h & =\text { molar residual enthalpy } & {[\mathrm{atm} \cdot l / \mathrm{mol}]} \\ K & =\text { vaporization equilibrium ratio } & {[-]} \\ k_{i j} & =\text { binary parameter for the } i-j \text { mixture } & {[-]} \\ P & =\text { pressure } & {[\mathrm{atm} \cdot l / \mathrm{mol} \cdot \mathrm{K}]} \\ R & =\text { gas constant } & {[\mathrm{K}]} \\ T & =\text { temperature } & {[l / \mathrm{mol}]} \\ v & =\text { molar volume } & {[-]} \\ Z & =\text { compressibility factor } & {[-]} \\ z & =\text { mole fraction } & \\ \eta & =\text { universal exponent in the mixing rule }\end{array}\right]$


$\begin{array}{ll}\phi & =\text { fugacity coefficient } \\ \omega & =\text { acentric factor }\end{array}$

$[-]$

$\langle$ Subscripts $\rangle$

$\begin{array}{ll}C M & =\text { pseudocritical property } \\ c & =\text { critical property } \\ M & =\text { mixture property } \\ r & =\text { reduced property } \\ i, j, k & =\text { component } i, j, k\end{array}$

〈Superscript >

(i) $\quad=$ component $i$

\section{Literature Cited}

1) Arai, K., H. Inomata and S. Saito: J. Chem. Eng. Japan, 15, 1 (1982).
2) Bender, E.: 5th International Congress of Chemical Engineering, CHISA 75, Section F2.25 (1975).

3) Joffe, J.: Ind. Eng. Chem., Fundam., 15, 298 (1976).

4) Lee, B. I. and M. G. Kesler: AIChE J., 21, 510 (1975).

5) Nishiumi, H. and S. Saito: J. Chem. Eng. Japan, 8, 356 (1975).

6) Plöcker, U., H. Knapp and J. Prausnitz: Ind. Eng. Chem., Process Des. Dev., 17, 324 (1978).

7) Reamer, H. H., R. H. Olds, B. H. Sage and W. N. Lacey: Ind. Eng. Chem., 34, 1536 (1942).

8) Soave, G.: Chem. Eng. Sci., 27, 1197 (1972).

9) Teja, A. S. and P. Rice: Chem. Eng. Sci., 36, 1 (1981).

10) Van Ness, H. C.: AIChE J., 1, 100 (1955).

11) Wiese, H. C., H. H. Reamer and B. H. Sage: J. Chem. Eng. Data, 15, $75(1970)$

\title{
LYSIS RATES OF FIBRIN CLOT BY PLASMIN
}

\author{
EIzo SADA, SHigeo KATOH AND SHUNiChIRo HARA \\ Department of Chemical Engineering, Kyoto University, Kyoto 606
}

Key Words: Medical Chemical Engineering, Biochemical Engineering, Enzyme Reaction, Adsorption, Fibrinolysis, Plasmin

\section{Introduction}

Under normal conditions a homeostatic balance between blood coagulation and fibrinolysis maintains fluidity in the vascular system. The conversion of fibrinogen to fibrin by thrombin makes a thrombus, as shown in Fig. 1. In the fibrinolytic system fibrin is hydrolyzed by the proteolytic enzyme plasmin, which is activated from plasminogen by plasmin activators. Therapeutic enhancement of the fibrinolytic system by these activators, such as urokinase and streptokinase, has been attempted for treatment of many pathological disorders caused by enhanced coagulability. Recent biochemical studies ${ }^{1)}$ of the plasminogen molecule elucidated that plasmin and plasminogen have the lysine-binding site and interact with fibrin and antiplasmin through it. The enzymatic lysis mechanism of solids such as fibrin is still uncertain. So it is interesting to study the effect of the interaction between plasmin or plasminogen and fibrin on the lysis rate. The present work was undertaken to measure the rates of fibrinolysis by plasmin and plasminogen-urokinase and to study the mechanism of fibrinolysis.

Received October 24, 1984. Correspondence concerning this article should be addressed to E. Sada.

\section{Materials and Methods}

Bovine serum fibrinogen (Nakarai Chemicals Co.) was made plasminogen-free by a lysine-Sepharose $4 \mathrm{~B}$ affinity column. Plasminogen and plasmin, which had the lysine-binding site, in bovine fibrinogen were selectively adsorbed in this column. The fibrinogen was fluoro-labelled by fluorescamine.

Bovine thrombin (587 NIH units/mg-protein, Sigma Chemicals Co.) was used to clot fibrinogen. Porcine plasmin and plasminogen (2.7 and 1.7 units/mg-protein, respectively, Sigma Chemicals Co.) were used and their activities were assayed by hydrolysis of casein. One unit is defined as $\Delta A_{280 \mathrm{~nm}}^{1 \mathrm{~cm}}=1.0$ for perchloric acid soluble products in $20 \mathrm{~min}$ at $\mathrm{pH}$ 7.5 and $37^{\circ} \mathrm{C}$. Plasminogen was activated by use of urokinase (Green Cross Co.).

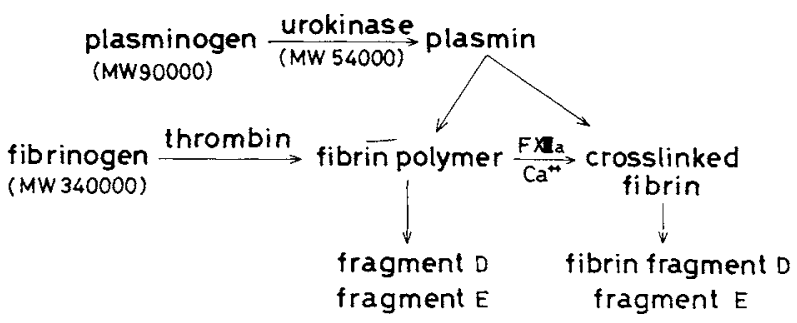

Fig. 1. Scheme of fibrinolysis. 